\title{
Are the Allergic Reactions of COVID-19 Vaccines Caused by mRNA Constructs or Nanocarriers? Immunological Insights
}

\author{
Gurudeeban Selvaraj $^{1}$ (1) Satyavani Kaliamurthi ${ }^{1}$ - Gilles H. Peslherbe ${ }^{1} \cdot$ Dong-Qing Wei $^{2,3}$
}

Received: 7 January 2021 / Revised: 29 April 2021 / Accepted: 7 May 2021 / Published online: 22 May 2021

(c) International Association of Scientists in the Interdisciplinary Areas 2021

\begin{abstract}
The Food and Drug Administration (FDA) has recently authorized the two messenger RNA (mRNA) vaccines BNT162b2 and mRNA-1273 for emergency use against the severe acute respiratory syndrome coronavirus 2 (SARS-CoV-2) causing the COVID-19 coronavirus disease. BNT162b2 and mRNA-1273 vaccines were developed by Pfizer-BioNTech and Moderna, respectively, in 2020. The United Kingdom, Bahrain, Canada, Mexico, United States, Singapore, Oman, Saudi Arabia, Kuwait, and European Union began their vaccination programs with the BNT162b2 vaccine, while the United States and Canada also started the mRNA-1273 vaccination program in mid December 2020. On 28th December 2020, studies reported severe allergic reactions in people who received the BNT162b2, and few people who received the mRNA-1273 vaccine. Authors of the letter thus attempt to explore possible causes of anaphylaxis following COVID-19 vaccination.
\end{abstract}

Keywords Anaphylaxis · Antibody $\cdot$ BNT162b2 $\cdot$ COVID-19 $\cdot$ IgE $\cdot$ MRNA-1273 $\cdot$ MRNA vaccine $\cdot$ SARS-CoV-2 $\cdot$ PEG

\section{Dear Editor,}

The Food and Drug Administration (FDA) recently authorized the two messenger RNA (mRNA) vaccines BNT162b2 and mRNA-1273 for emergency use against the severe acute respiratory syndrome coronavirus 2 (SARS-CoV-2) causing the COVID-19 coronavirus disease. BNT162b2 and mRNA-1273 vaccines were developed by Pfizer-BioNTech and Moderna, respectively, in 2020. The United Kingdom, Bahrain, Canada, Mexico, United States, Singapore, Oman, Saudi Arabia, Kuwait, and European Union began their

Gurudeeban Selvaraj

gurudeeban.selvaraj@ concordia.ca

$\triangle$ Dong-Qing Wei

dqwei@sjtu.edu.cn

1 Centre for Research in Molecular Modeling, Department of Chemistry and Biochemistry, Concordia University, 7141 Sherbrooke Street W, Montreal, QC H4B1R6, Canada

2 The State Key Laboratory of Microbial Metabolism, College of Life Sciences and Biotechnology, Shanghai Jiao Tong University, No. 800 Dongchuan Road, Minhang, Shanghai 200240, China

3 IASIA (International Association of Scientists in the Interdisciplinary Areas), 125 Boul. de Bromont, Quebec, QC J2L 2K7, Canada vaccination programs with the BNT162b2 vaccine, while the United States and Canada also started the mRNA-1273 vaccination program in mid December 2020.

In the last 2 weeks, we read about the components of these two mRNA vaccines from different media resources. Briefly, the BNT162b2 vaccine contains the spike glycoprotein of SARS-CoV-2 coding mRNA, 2 [polyethylene glycol (PEG)-2000]- $N$, $N$-di-tetradecyl acetamide, 1, 2-distearoylsn-glycero-3-phosphocholine, cholesterol, (4-hydroxybutyl) azanediyl) bis (hexane-6, 1-diyl) bis (2-hexyldecanoate), potassium chloride, monobasic potassium phosphate, sodium chloride, dibasic sodium phosphate dehydrate, and sucrose. COVID-19 patients or volunteers (age $>16$ ) who received BNT162b2 vaccination at 21-day intervals of two dosages (30 $\mu \mathrm{g} / \mathrm{dose})$ attained $95 \%$ protection (ClinicalTrials.gov No: NCT04368728) against SARS-CoV-2 [1]. Likewise, mRNA-1273 vaccine components include the spike glycoprotein of SARS-CoV-2 coding mRNA, PEG 2000 dimyristoyl glycerol, 1,2-distearoyl-sn-glycero3-phosphocholine, cholesterol, SM-102, tromethamine, tromethamine hydrochloride, acetic acid, sodium acetate, and sucrose. Vaccinated COVID-19 patients or volunteers (age $>18)$ with mRNA-1273 $(2 \times 100 \mu \mathrm{g} /$ dose $)$ in 28 days of interval acquired $94.1 \%$ protection (ClinicalTrials.gov No: NCT04470427) against SARS-CoV-2 [2]. The incidence of serious adverse effects of these mRNA vaccines 
was reported low but included mild-to-moderate pain at the injection site, fatigue, and headache. Both candidate vaccines use PEGlyated lipid nanoparticles as a carrier for vaccine delivery, which is administered through intramuscular injection to the patients and volunteers as well.

The demographic and clinical characteristics of the mRNA-1273 vaccine included participants (0.6-4.9\%) having a history of chronic lung disease, cardiac disease, severe obesity, diabetes, liver disease, and human immunodeficiency virus infection. Moreover, the clinical trials of the BNT162b2 vaccine were conducted with a random selection of participants. On 28th December 2020, Banerji et al. [3] reported severe allergic reactions in some people who received the BNT162b2, and fewer people who received the mRNA-1273 vaccine. In addition, Castells and Phillips [4] noted that severe anaphylaxis reaction was observed in health care workers within $24 \mathrm{~h}$ after vaccination in the United Kingdom and Boston, United States [5]. Thus, the National center for immunization and respiratory diseases (NCIRD), Centers for disease control and prevention (CDC) advised health authorities of all countries to exclude anyone with a history of a severe allergic reaction associated with one of the vaccine components, especially PEG and PEG derivatives like polysorbates $[6,7]$.

Notably, allergens are mostly the proteins or glycoproteins or excipients present in the therapeutic molecules (e.g., vaccines) that promote or stimulate allergen-specific antibodies (i.e., immunoglobulins type $\mathrm{E}$ or $\mathrm{IgE}$ ) in the human body [8]. These allergens react with $\operatorname{IgE}$ and cause allergic inflammatory responses. The crosslinking of $\operatorname{IgE}$ antibodies that are bound to the Fc epsilon RI (high-affinity IgE receptor) on the basophils and mast cells triggers degranulation in a short period. It results in the release of inflammatory mediators, namely histamine, prostaglandins and leukotrienes, proteases (G-protein-coupled receptors), and pro-inflammatory cytokines; and observation of major allergic symptoms (e.g., nausea, vomiting, reddening, rashes, laryngeal edema, wheezing, tachycardia, hypotension, and cardiovascular collapse) [8].

However, scientific personnel and medical professionals need to determine and understand the possible cause of allergic reactions, whether it by antigenic fragments or epitope (i.e., translated spike glycoprotein fragments) or excipients or stabilizer (PEG) in the vaccine molecules.

Glycoprotein-induced anaphylaxis: Many food allergens include egg, fish, milk, shellfish, and tree nuts that contain water-soluble glycoproteins, which are relatively stable to heat, acid, and proteolytic action $[9,10]$. To provoke sustained immune response, the immunological (glycoprotein) molecule that binds specifically with the membrane receptor (i.e., antibody IgE) on T or B cell is known as an epitope. This epitope may be a linear (stable) or conformational (less stable) epitope. The linear epitopes are formed by the three-dimensional conformation adopted by the IgE paratope interaction of contiguous amino acid residues. Then, the IgE-allergen complex that binds with the receptor on mast cells or basophils causes allergic reactions such as the release of histamine, prostaglandins, and leukotrienes, proteases [11]. From the immunological point of view, after vaccination, these mRNA vaccines enter into the human cells and are translated into protein fragments (especially spike glycoprotein), which they present to the surface and are recognized by the human immune cells (e.g., APCantigen-presenting cells). The APC present these spike protein fragments (epitopes) through MHC-II molecules on its surface. Further, the MHC-II-epitope complex was identified by the $\mathrm{T}$ helper cell receptor, which can raise the alarm and trigger the $\mathrm{T}$ helper cells-mediated immune response to release interleukins [12]. It involves the activation of allergen-specific B cells and the production of IgE antibodies (Fig. 1). Notably, nucleoside-modified mRNA (BNT162b2 and mRNA-1273) translated into fragments of spike glycoprotein of SARS-CoV-2, which possibly contains water-soluble glycoprotein fragments (allergen), triggers anaphylaxis reaction in some of the vaccinated individuals.

Stabilizer-induced anaphylaxis: BNT162b2 and mRNA1273 vaccines contain two different stabilizing agents such as sucrose and PEG. In general, carbohydrate (sucrose) intolerance does not disturb the immune system and it may cause common allergic reactions, namely gastrointestinal disturbance. Another stabilizer PEG is non-toxic approved by the FDA in 1990. PEG is commonly used for surface components of lipid nanoparticles reported to have higher stability, circulation half-life, and reduced immunogenicity [13]. In general, mRNAs are short and easily degraded by the natural enzymes in the human body. Thus, the two vaccines included PEG-lipid nanoparticles coating to protect the mRNA in the circulation in the human body. Besides, studies of PEG encapsulated nanomedicine reportedly can cause pseudo-allergic reactions in experimental models [14, 15]. Similarly, after endocytosis, the mRNA free PEG in lipid nanoparticles floating on the cell surface interact with IgE antibodies (i.e. allergen) that bind into the FcER1 receptor on mast cells or basophils and can trigger immediate release of inflammatory and pro-inflammatory mediators that include histamine, prostaglandins, and proteases. Usually, the anaphylaxis signs and symptoms appear within a few minutes to one hour after exposure to allergic substances or antigens in the vaccine (Fig. 1).

The spike glycoprotein of SARS-CoV-2 contains key regions that include the receptor-binding domain (RBD), integrin-binding motif, an RB motif that binds to human angiotensin-converting enzyme 2 (ACE2B), two fusion peptides, and two heptad repeats. The spike glycoprotein (P0DTC2) sequence was retrieved from the Uniprot database. Allergenicity of the spike protein key regions was 


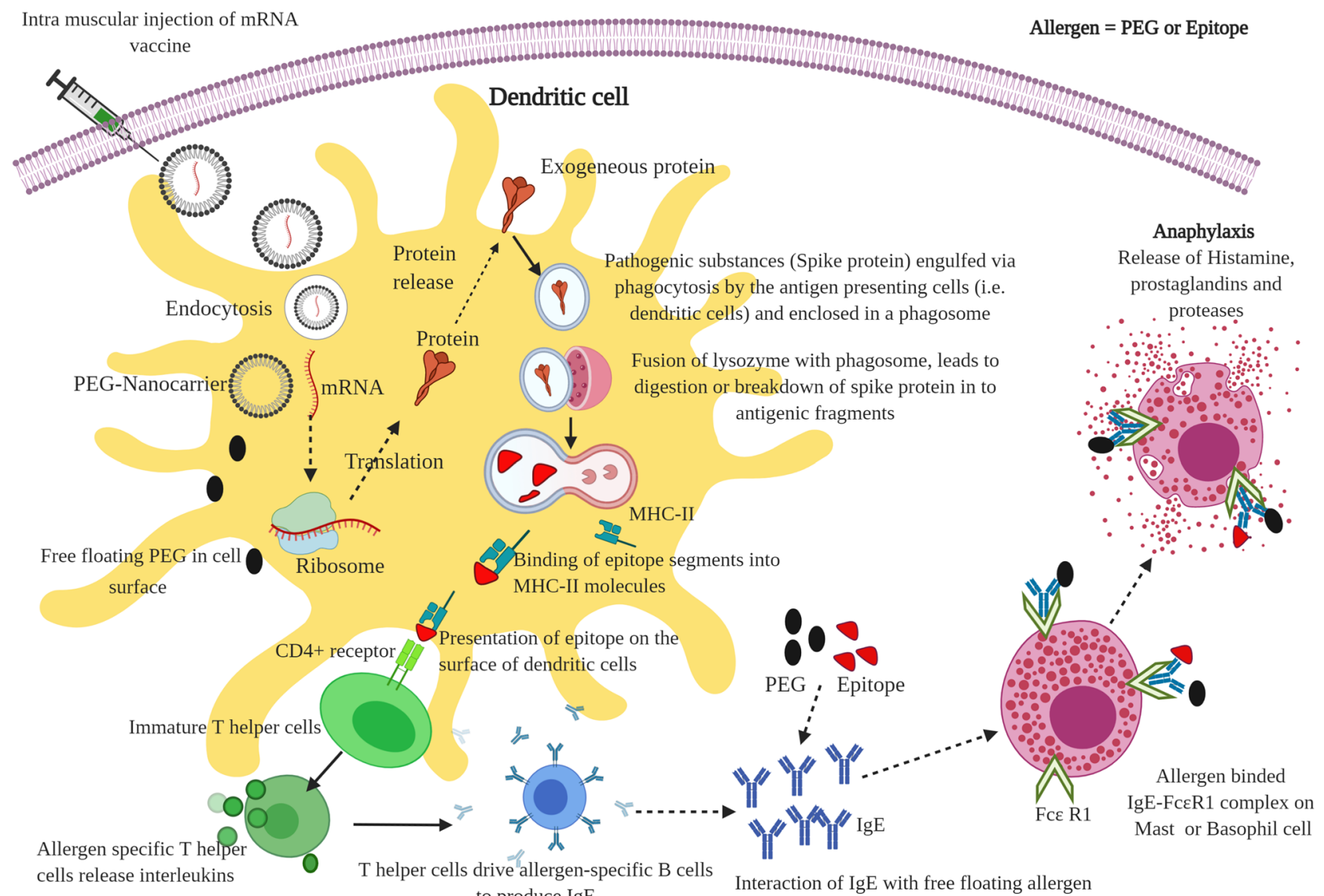
to produce IgE

Fig. 1 Schematic representation of possible mechanism of anaphylaxis reaction after mRNA vaccination for COVID-19

computationally predicted using two different tools AllerTOP v. 2.0 and AllergenFP v.1.0, which are effectively used for subunit vaccine design $[16,17]$. The results from AllerTOP indicate that the RBD, receptor-binding motif, binding to human ACE2B (437-508), and two fusion peptides could be a probable allergen, while the results from both tools point to the receptor-binding motif of the spike protein to the host receptor ACE2B as a very probable allergen (Table 1). These results suggest that spike protein-based medication possibly induces an allergic reaction in the host system, likely due to the amino acid residues 437-508 sequence.
Moreover, spike protein key substitutions (L452R and $\mathrm{E} 484 \mathrm{~K}$ ) have been reported in identified variants of SARS-CoV-2. The L452R substitutions were reported in B.1.526.1, B.1.427, and B.1.429 variants and E484K reported in variants B.1.525, P.2, P.1, and B.1.351 (large number of patients) and B.1.526 and B.1.1.7 (minimal number of patients) [18]. These reports indicate that amino acid residues (437-508) of the spike protein may cause possible anaphylaxis, which needs to be further investigated in COVID-19 prophylactic or therapeutic drug or vaccine development.
Table 1 Allergenicity prediction of key regions of spike glycoprotein of SARS-CoV-2

\begin{tabular}{lcll}
\hline Key regions of spike glycoprotein of SARS-CoV-2 & Position (s) & AllerTOP v. 2.0 & AllergenFP v.1.0 \\
\hline Receptor-binding domain (RBD) & $319-541$ & Allergen & Non-allergen \\
Integrin-binding motif & $403-405$ & Non-allergen & Non-allergen \\
Receptor-binding motif, binding to human ACE2B & $437-508$ & Allergen & Allergen \\
Fusion peptide 1 & $816-837$ & Allergen & Non-allergen \\
Fusion peptide 2 & $835-855$ & Allergen & Non-allergen \\
Heptad repeat 1 & $920-970$ & Non-allergen & Non-allergen \\
Heptad repeat 2 & $1163-1202$ & Non-allergen & Non-allergen \\
\hline
\end{tabular}


Further, the cohort study by the University of Oxford reported that two mRNA vaccines (BNT162b2 and mRNA1273 ) and the AstraZeneca vaccine possibly cause cerebral venous thrombosis (i.e., anaphylaxis reaction) after 14 days of vaccination [19]. The mechanism of action was not reported in the article but there is a chance that spike proteins may elicit anaphylaxis reaction because of the major similarity of these vaccines is the presence of "spike protein immunogen".

At present, vaccination programs have been initiated concurrently against COVID-19, but the debate is still going about the adverse effects and safety concerns raised by the vaccine. Moreover, the allergic reactions of the participants need to be identified or screened frequently by questionnaires and IgE testing before and after vaccination to monitor unpleasant adverse effects of the vaccine. In this concern, promising research outputs are needed to confirm the safety of the currently formulated mRNA vaccines.

Acknowledgements Partial support of this work was provided by the Natural Sciences and Engineering Research Council (NSERC) of Canada (G.H.P. - Grant no. 216940); National Natural Science Foundation of China (DQ.W. -Grant no. 61832019, 61503244), the Ministry of Science and Technology of China (DQ.W.-Grant no.: 2016YFA0501703); G.S. and S.K. are grateful to the MITACS Global Research award, Concordia University for bridge funding from the Faculty of Arts and Science, the Office of the Vice-President Research and Graduate Studies, and Concordia International.

\section{Declarations}

Conflict of Interest All authors declared there is no conflict of interest.

\section{References}

1. Polack FP, Thomas SJ, Kitchin N, Absalon J, Gurtman A, Lockhart S, Perez JL, Pérez Marc G, Moreira ED, Zerbini C, Bailey R (2020) Safety and efficacy of the BNT162b2 mRNA COVID-19 vaccine. N Engl J Med 383:2603-2615. https://doi.org/10.1056/ NEJMoa2034577

2. Baden LR, El Sahly HM, Essink B, Kotloff K, Frey S, Novak R, Diemert D, Spector SA, Rouphael N, Creech CB, McGettigan J (2020) Efficacy and safety of the mRNA-1273 SARS-CoV-2 vaccine. N Engl J Med. https://doi.org/10.1056/NEJMoa2035389 (in press)

3. Banerji A, Wickner PG, Saff R, Stone CA Jr, Robinson LB, Long AA, Wolfson AR, Williams P, Khan DA, Phillips E, Blumenthal KG (2020) mRNA vaccines to prevent COVID-19 disease and reported allergic reactions: current evidence and approach. J Allergy Clin Immunol. https://doi.org/10.1016/j.jaip.2020.12.047 (in press)

4. Castells MC, Phillips EJ (2020) Maintaining safety with SARSCoV-2 vaccines. N Engl J Med. https://doi.org/10.1056/NEJMr a2035343 (in press)
5. Cabanillas B, Akdis C, Novak N (2020) Allergic reactions to the first COVID-19 vaccine: a potential role of Polyethylene glycol? Allergy. https://doi.org/10.1111/all.14711 (in press)

6. NCIRD (National Center for Immunization and Respiratory Diseases) (2020a) Interim Clinical Considerations for Use of mRNA COVID-19 Vaccines Currently Authorized in the United States. Released on December 30, 2020 https://www.cdc.gov/vaccines/ covid-19/info-by-product/clinical-considerations.html

7. NCIRD (National Center for Immunization and Respiratory Diseases) (2020b) COVID-19 Vaccines and Allergic Reactions. Released on December 31, 2020 https://www.cdc.gov/coronavirus/2019-ncov/vaccines/safety/allergic-reaction.html

8. Aalberse RC (2000) Structural biology of allergens. J Allergy Clin Immunol 106(2):228-238. https://doi.org/10.1067/mai.2000. 108434

9. Radauer C, Breiteneder H (2007) Evolutionary biology of plant food allergens. J Allergy Clin Immunol 120(3):518-525. https:// doi.org/10.1016/j.jaci.2007.07.024

10. Cianferoni A, Muraro A (2012) Food-induced anaphylaxis. Immunol Allergy Clin North Am 32(1):165-195. https://doi.org/10. 1016/j.iac.2011.10.002

11. Valenta R (2008) Biochemistry of allergens and recombinant allergens. In: Kay AB (ed) Allergy and allergic diseases. Blackwell Publishing Ltd, Oxford, pp 895-912. https://doi.org/10.1002/ 9781444300918.ch42

12. Janeway CA Jr, Travers P, Walport M, Shlomchik MJ (2001) Immunobiology: the immune system in health and disease, 5th edn. Garland Science, New York https://www.ncbi.nlm.nih.gov/ books/NBK10757/

13. Ryals RC, Patel S, Acosta C, McKinney M, Pennesi ME, Sahay G (2020) The effects of PEGylation on LNP based mRNA delivery to the eye. PLoS ONE 15(10):e0241006. https://doi.org/10.1371/ journal.pone.0241006

14. Kozma GT, Mészáros T, Vashegyi I, Fülöp T, Örfi E, Dézsi L, Rosivall L, Bavli Y, Urbanics R, Mollnes TE, Barenholz Y (2019) Pseudo-anaphylaxis to polyethylene glycol (PEG)-coated liposomes: roles of anti-PEG IgM and complement activation in a porcine model of human infusion reactions. ACS Nano 13(8):9315-9324. https://doi.org/10.1021/acsnano.9b03942

15. Sellaturay P, Nasser S, Ewan P (2020) Polyethylene glycolinduced systemic allergic reactions (anaphylaxis). J Allergy Clin Immunol. https://doi.org/10.1016/j.jaip.2020.09.029 (in press)

16. Dimitrov I, Bangov I, Flower DR, Doytchinova I (2014) AllerTOP vol 2-a server for in silico prediction of allergens. J Mol Model 20:2278. https://doi.org/10.1007/s00894-014-2278-5

17. Dimitrov I, Naneva L, Doytchinova I, Bangov I (2014) Allergen FP: allergenicity prediction by descriptor fingerprints. Bioinformatics 30:846-851. https://doi.org/10.1093/bioinformatics/btt619

18. NCIRD (National Center for Immunization and Respiratory Diseases) (2021) SARS-CoV-2 Variant Classifications and Definitions. Released on April 27, 2021 https://www.cdc.gov/coron avirus/2019-ncov/cases-updates/variant-surveillance/variant-info. html

19. Taquet M, Husain M, Geddes JR, Luciano S, Harrison PJ (2021) Cerebral venous thrombosis and portal vein thrombosis: a retrospective cohort study of 537,913COVID-19 cases. Open Sci Framew. https://doi.org/10.17605/OSF.IO/H2MT7 